\title{
Bradycardia, Hypotension, and Midventricular Takotsubo Syndrome during Esophagogastroduodenoscopy
}

To the Editor:

The two cases of women in their mid-40s who experienced Takotsubo syndrome (TTS) in the setting of esophagogastroduodenoscopy (EGD), reported by Yu et al. ${ }^{1}$ in the January issue of Clinical Endoscopy, are interesting because the patients were premenopausal, had midventricular TTS, were under conscious sedation, and did not appear to be under stress during the procedure. The first patient initially had hypertension and tachycardia, which was followed by hypotension and bradycardia, suggesting vagotonia, a feature characteristic of the later presentation of TTS. ${ }^{2,3}$ The other patient developed hypotension and tachycardia "1 minute after the insertion of the EGD scope." One wonders whether TTS in the setting of EGD is mediated by intense vagotonia, characterized by hypotension and/or bradycardia. ${ }^{2,3}$ with tachycardia and/or hypertension being mediated by a reflex counterbalancing intense activation of the autonomic sympathetic nervous system, and the effects of the drugs administered to manage the hypotension and/or bradycardia.

The authors refer to five cases that have been reported worldwide to be associated with gastrointestinal endoscopy, ${ }^{1}$ and state that "previous reports indicated one case of EGD in a postmenopausal woman with sedation, three cases of colonoscopy (COL), and one case of simultaneous EGD and COL in a postmenopausal woman., ${ }^{31,4-6}$ I was able to access the paper describing the patient with both EGD and COL, who had TTS complicated by ventricular fibrillation, but I could not access the other two papers the authors are referring to. ${ }^{5,6}$ I would appreciate comments by the authors on the remaining four patients with TTS, including the circumstances under which they experienced TTS, their ages and sex, whether they were postmenopausal, had hypotension and/or bradycardia, and the type of TTS they developed (apical, midventricular, basal, or segmental).

\section{Conflicts of Interest}

The author has no financial conflicts of interest.

John E. Madias

Division of Cardiology, Elmhurst Hospital Center, Icahn School of Medicine at Mount Sinai, Elmhurst, NY, USA

\section{REFERENCES}

1. Yu JW, Park J, Song PS, et al. Two cases of stress cardiomyopathy during esophagogastroduodenoscopy. Clin Endosc 2016;49:76-80.

2. Samuels MA. Neurally induced cardiac damage. Definition of the problem. Neurol Clin 1993;11:273-292.

3. Samuels MA. The brain-heart connection. Circulation 2007;116:77-84.

4. Kim SR, Nakashima K, Nishiuchi S, et al. A case of takotsubo cardiomyopathy with ventricular fibrillation after gastroenterological endoscopy. Clin J Gastroenterol 2011;4:73-78.

5. Akashi R, Oogushi M, Yoshida M, et al. A case report of "takotsubo" type cardiomyophaty during upper digestive endoscopy. Gastroenterol Endosc 2003;45:253-260.

6. Kaneko E, Harada H, Kasugai T, Ogoshi K, Tanba H. Complications from gastrointestinal endoscopy procedure from 1998 to 2002. Gastroenterol Endosc 2004;46:54-61. 


\section{RESPONSE}

Thank you for your interest in our case report.

As you know, stress cardiomyopathy associated with gastrointestinal endoscopic procedures is very rare. On a literature review, we found just five cases. A case report on stress cardiomyopathy with ventricular fibrillation associated with esophagogastroduodenoscopy and colonoscopy can be accessed in English. ${ }^{1}$ Unfortunately, the other two reports published by the Japan Gastroenterological Endoscopy Society are written in Japanese, but the abstract and figures are available in English. $^{2,3}$

In the case reported by Akashi et al., ${ }^{2}$ the patient was a 74-year-old woman with hypertension. Bradycardia was noted at the time of scope insertion. The transthoracic echocardiogram showed akinesis of the anterior wall, septum, and part of the posterior wall in the apex of the left ventricle; these had fully recovered by the next day. Myocardial scintigraphy demonstrated low radioisotope uptake in the anterior and apical regions of the cardiac muscle, but it returned to normal by the next day.

The report by Kaneko et al. ${ }^{3}$ was a Japanese nationwide review of complications associated with gastrointestinal endoscopic procedures from 1998 to 2002. Therefore, the case-spe- cific data were not available.

For more information, please connect to the web links: Akashi et al. ${ }^{2}$ (https://www.jstage.jst.go.jp/article/ gee1973b/45/3/45_3_253/_article) and Kaneko et al. ${ }^{3}$ (https:// www.jstage.jst.go.jp/article/gee1973b/46/1/46_1_54/_article/-char/ja/).

\section{Conflicts of Interest}

The authors have no financial conflicts of interest.

Jong Won Yu and Jongha Park

Department of Internal Medicine, Inje University Haeundae Paik Hospital, Inje University College of Medicine, Busan, Korea

\section{REFERENCES}

1. Kim SR, Nakashima K, Nishiuchi S, et al. A case of takotsubo cardiomyopathy with ventricular fibrillation after gastroenterological endoscopy. Clin J Gastroenterol 2011;4:73-78.

2. Akashi R, Oogushi M, Yoshida M, et al. A case report of "takotsubo" type cardiomyophaty during upper digestive endoscopy. Gastroenterol Endosc 2003;45:253-260.

3. Kaneko E, Harada H, Kasugai T, Ogoshi K, Tanba H. Complications from gastrointestinal endoscopy procedure from 1998 to 2002. Gastroenterol Endosc 2004;46:54-61. 